\title{
AN ACCURATE MODEL OF A FULL OPTICAL ENCODER/DECODER IN A WGR CONFIGURATION
}

\author{
Gabriella Cincotti ${ }^{1}$, N. Wada ${ }^{2}$, and K. Kitayama ${ }^{3}$ \\ 'Department of Applied Electronics, University of Roma Tre, via della Vasca Navale 84, \\ I-00146 Rome, Italy, g.cincotti@uniroma3.it \\ ${ }^{2}$ National Institute of Information and Communications Technology \\ Tokio,Japan,wada@nict.go.jp \\ ${ }^{3}$ Department of Electronic and Information Systems, Osaka University, 2-1 Yamadaoka, Suita, \\ Osaka565-0871, Japan, kitayama@comm.eng.osaka-u.ac.jp \\ Abstract: We present a field model of a full Encoder/Decoder (E/D) in a Waveguide \\ Grating Router (WGR) configuration that generates/processes a set of Phase- \\ Shift Keyed (PSK) codes. We furnish the design procedure to synthesize the \\ device, determining the optimal number of the ports and the tolerance in the \\ differential path length as functions of the code detection parameter.
}

\section{INTRODUCTION}

In Refs. [1-3], we presented innovative planar architectures for a full optical Encoder/Decoder (E/D), that is passive optical devices with a single input and $N$ outputs to generate/process $N$ PSK codes simultaneously. In particular, we showed that the standard Waveguide Grating Router (WGR) configuration of Fig. 1 can be synthesized as a full E/D: if a single optical pulse is directed into one of the device inputs, at the device outputs we obtain $N$ PSK codes. On the other hand, if one PSK code is forwarded into the same device input, at the device outputs we obtain the correlation functions between the input code an all the PSK codes in the look-up table.

WGRs are recognized as key passive components in current optical communication systems and they have been proposed to implement a large variety of applications, as dispersion compensation, frequency demultiplexers, tuneable filters and optical signal processing [4-7]. The transmission function of WGR 
frequency demultiplexers has been largely investigated, performing the spectral analysis by means of the Fourier optics $[8,9]$.

But the mechanism to build a set of PSK codes can be more easily described analyzing the WGR configuration in the time domain: the input slab coupler generates $N$ copies of the input pulse, with phases given by the Rowland circle configuration [4]. The optical pulses travel different paths in the Arrayed Waveguides (AW) grating and the output slab coupler recombines the pulses to built $N$ codes at the device outputs; each PSK code is composed of $N$ optical pulses, which are often referred to as chips. The differential path delay $\Delta \tau$ in the $\mathrm{AW}$ grating is chosen smaller than the input pulse width $\delta_{l}$, so that the chips in the output codes do not overlap. It is evident that the design requirements of a full E/D and a standard demultiplexer are greatly different: in the present paper we furnish an accurate model for the full $\mathrm{E} / \mathrm{D}$, along with the corresponding design guidelines.

The remainder of the paper is organized as follows: in Sec. 2, we give a very simple but comprehensive field model of the E/D, and introduce the PSK codes detection parameter. In Sec. 3, we evaluate the optimal number of ports and the fabrication tolerance of the proposed device architecture, as functions of the detection parameter. Conclusions and hints for future works are given in the last section.

\section{FIELD MODEL}

We refer to the device architecture of Fig. 1 and the parameters listed in Table I, and evaluate the impulse response from the $i$-th input to the $k$-th output as

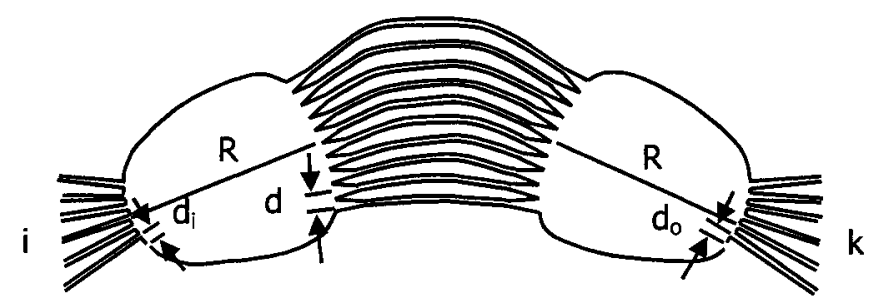

Figure 1. Geometry of the E/D in the WGR configuration. 
Table 1. Device parameters

\begin{tabular}{lll}
\hline parameter & description & value \\
\hline$f_{0}$ & carrier frequency $\left(\lambda_{0}=1550 \mathrm{~nm}\right)$ & $193.55 \mathrm{THz}$ \\
$N$ & number of the AW grating arms & 16 \\
$R$ & input/output slab focal length & $20.85 \mathrm{~mm}$ \\
$d$ & spacing in the AW grating & $24.6 \mu \mathrm{m}$ \\
$d_{i}, d_{o}$ & waveguide spacing in the input/output grating & $56.47 \mu \mathrm{m}$ \\
$L$ & shortest waveguide length & $26 \mathrm{~mm}$ \\
$\Delta L$ & differential path length & $1.0316 \mathrm{~mm}$ \\
$n_{e}$ & refractive index in the AW grating & 1.454 \\
$n_{s}$ & refractive index in the slab regions & 1.451 \\
\hline
\end{tabular}

$$
h_{i k}(t)=\sum_{j=0}^{N-1} \delta\left(t-\frac{n_{e}(L+j \Delta L)}{c}\right) e^{-j 2 \pi j\left[\frac{n_{s} d}{\lambda_{0} R}\left(i d_{i}+k d_{o}\right)\right]},
$$

where $\delta(t)$ is the delta function, $j$ the imaginary unit and $\Delta \tau=n_{e} \Delta L / c$ is the chips interval. Assuming that the input and the output waveguide gratings have the same spacing, i.e. $d_{i}=d_{o}$, we set $\lambda_{0} R /\left(d d_{o} n_{S}\right)=N$; in this case, the transfer function from the $i$-th input to the $k$-th output is

$$
\begin{aligned}
& H_{i k}(f)=e^{-j 2 \pi \frac{n_{e} L}{c} f} \sum_{j=0}^{N-1} e^{-j 2 \pi j\left(\frac{i+k}{N}+\Delta \tau f\right)} \\
& =e^{-j 2 \pi \frac{n_{e} L}{c} f} e^{-j \pi\left(\frac{i+k}{N}+\Delta \tau f\right)(N-1) \sin [\pi(i+k+N \Delta \tau f)]}
\end{aligned}
$$

that is plotted in Fig. 2. The code generated are highly orthogonal and their crosscorrelation is almost zero everywhere; in fact, according to the Wiener theorem, the cross-correlation function between the two PSK codes at the outputs $k$ and $k^{\prime}$ can be evaluated as [10]

$$
h_{i k}(t) * h_{i k}(t)=\int_{-\infty}^{\infty} H_{i k}(f) H_{i k}(f) e^{i 2 \pi f t} d f
$$




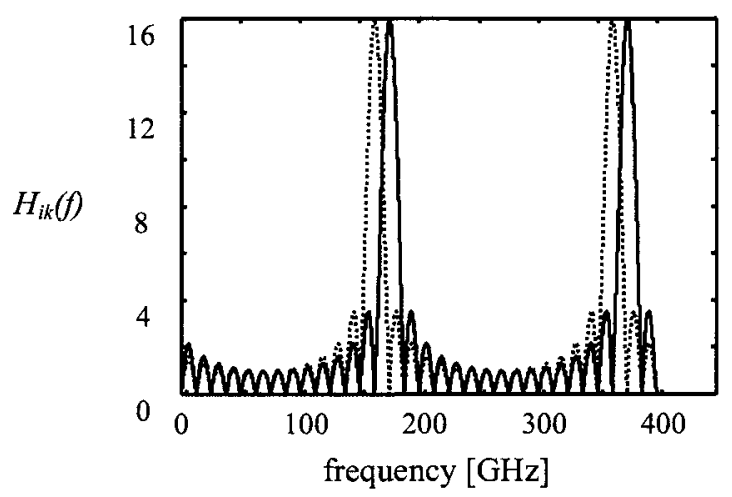

Figure 2. Transfer function at two adjacent outputs.

Since the transfer functions at two different outputs do not overlap (see Fig. 2), the Cross-Correlation Peak (CCP) is very small.

The Auto-Correlation Peak (ACP) detected at one of the device outputs reveals an exact match between the incoming PSK code and the corresponding code in the look up table; the parameter that characterizes the code detectability is the ratio $r=\mathrm{ACP} / \mathrm{CCP}$. It is quite immediate verify that the $\mathrm{ACP}=N^{2}$, whereas the CCP depends on the two outputs $k$ and $k^{\prime}$. From an inspection of Eq. (2), it is evident that the CCP is higher for two PSK codes generated at two adjacent outputs, and in the followings we evaluate the $r$ parameter in this worst case condition. We remark that lower values of the $r$ parameter correspond to outputs that are spatially adjacent, not because the crosstalk, but only because the codes generated at adjacent outputs are more correlated.

To numerically investigate the performances of the proposed PSK codes set, we consider a Gaussian optical pulses of width $\delta_{l}=2 \mathrm{ps}$

$$
p(t)=e^{-\frac{t^{2}}{2 \delta_{t}^{2}}} e^{-i 2 \pi f_{0} t}
$$

at one of the device inputs, that generates $N=16$ PSK codes, each of them composed of $N=16$ chips. Setting the chip interval $\Delta \tau=10 \mathrm{ps}$, we obtain the PSK codes of Fig. 3, with $\mathrm{ACP}=256, \mathrm{CCP}=26.27$ and $r=9.74$.

Quite similar results are obtained decreasing the chip interval: in the case of $\Delta \tau=5 \mathrm{ps}$, we have $\mathrm{ACP}=296.43, \mathrm{CCP}=30.9$ and $r=9.74$, as shown in Fig. 4 . 
470
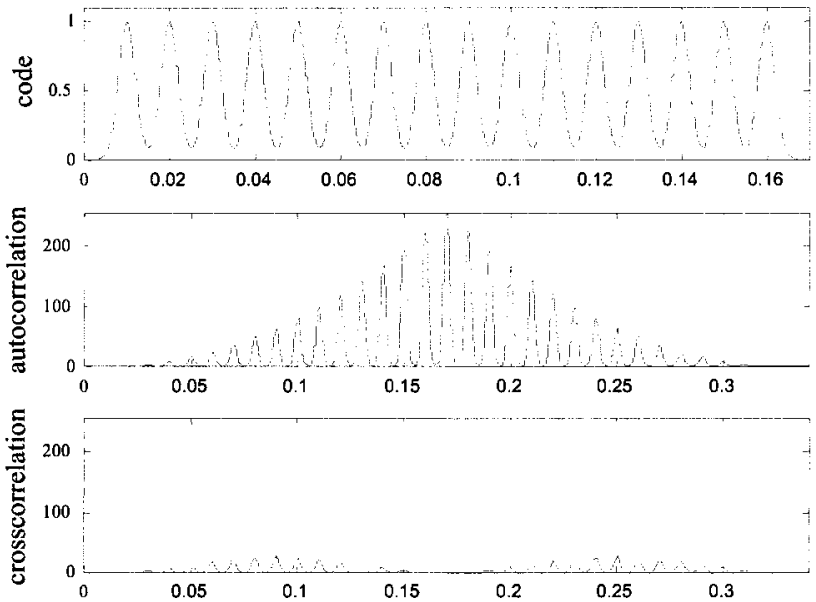

Figure 3: PSK code composed of $N=16$ Gaussian chips, of width $\delta_{l}=2 p s$ and chip interval $\Delta \tau=10 p s$.

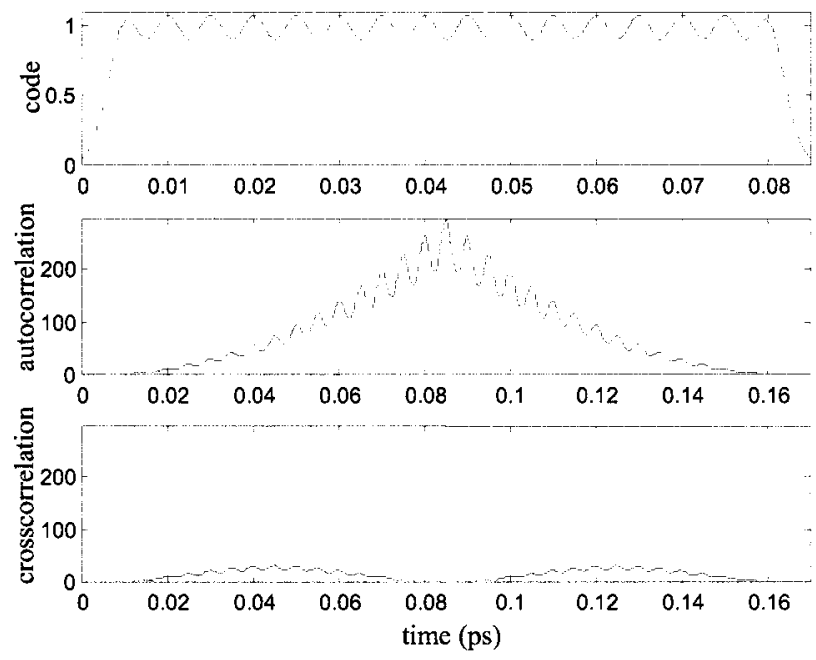

Figure 4: PSK code composed of $N=16$ Gaussian chips, of width $\delta_{i}=2 p s$ and chip interval $\Delta \tau=5 p s$ 


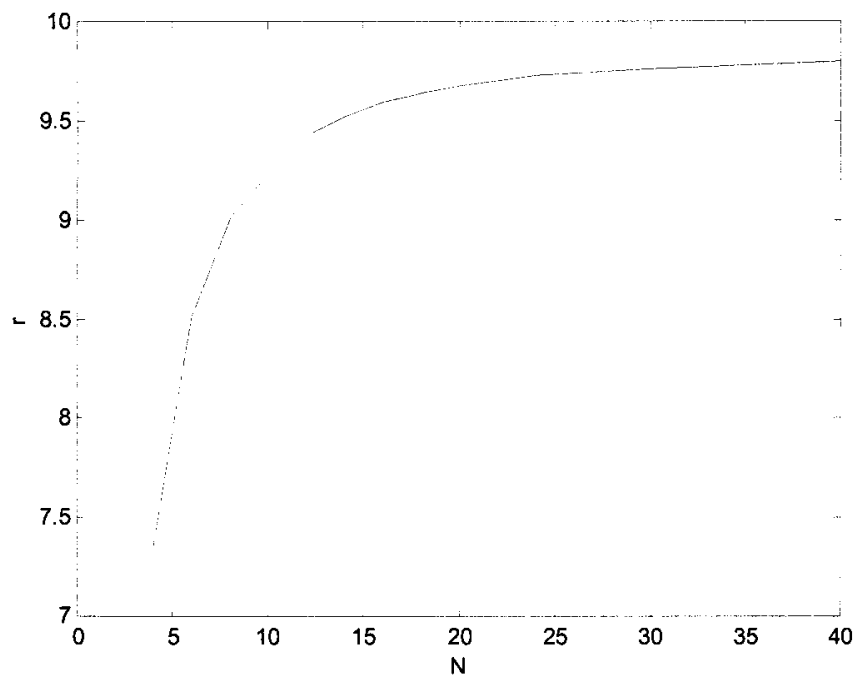

Figure 5: Label detection parameter $r=\mathrm{ACP} / \mathrm{CCP}$ versus the number of the outputs $N$.

\section{DESIGN EQUATIONS}

The number $N$ of the device inputs/outputs coincides with both the number of the PSK codes and the code length, i.e. the number of the chips in every code. Therefore, we have to trade the code cardinality, i.e. the number of PSK codes that we can simultaneously generate and process, for the code processing time, i.e. the time necessary to perform the correlations that is $(N-1) \Delta \tau$ [11]. In the case $N=16$, the code processing speed is $1 /(N-1) \Delta \tau=13.310^{9} \mathrm{pps}$.

The ACP coincides with $N^{2}$, and to increase the code detection parameter $r=\mathrm{ACP} / \mathrm{CCP}$, we should consider a high value of $N$. In Fig. 5 we plot the $r$ parameter for two adjacent outputs as function of $N$ : it is evident that the $r$ parameter increases with $N$ and that there is a saturation behaviour for $N>25$.

The main parameter of the WGR architecture is the differential path length $\Delta L$, that determines the chip interval. The tolerance of this parameter depends on the input pulse width $\delta_{t}$ and the chip interval $\Delta \tau$. Fig. 6 shows the $r$ parameter as a function of the differential path length variation, when all the path lengths in the AW grating increase $(+)$ or decrease $(-)$ of the same value.

The other device parameters has to be chosen so that $\lambda_{0} R /\left(d d_{o} n_{S}\right)=N$, to set the phases of the chips. Therefore, any change in the device parameters $d, d_{o}, R$, $n_{s}$ or the reference wavelength $\lambda_{0}$ causes a change in the code phases. However, their change slightly influences the device performances, as shown in Fig 7. 
472

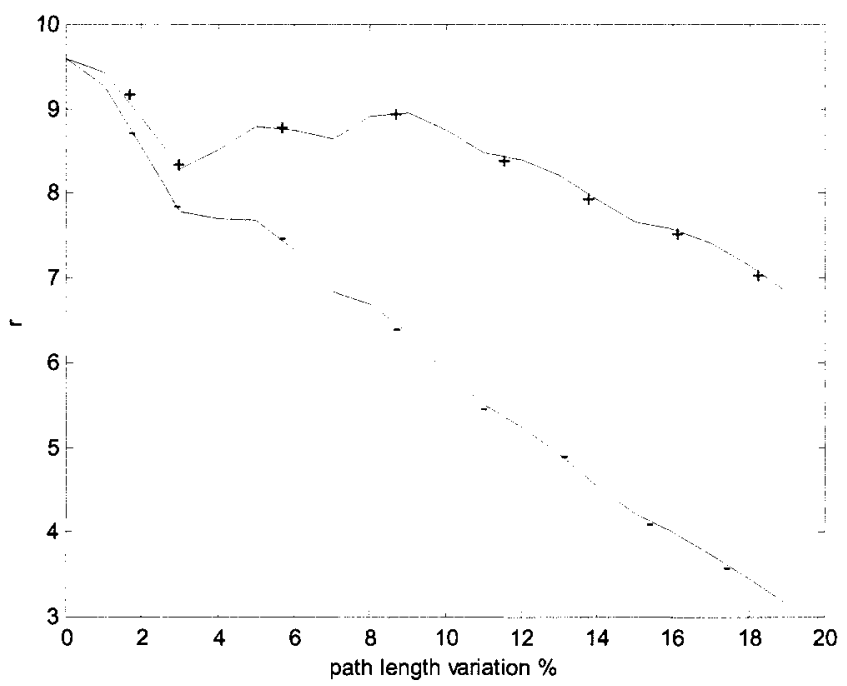

Figure 6. Code detection parameter $r$ versus the differential path length variation.

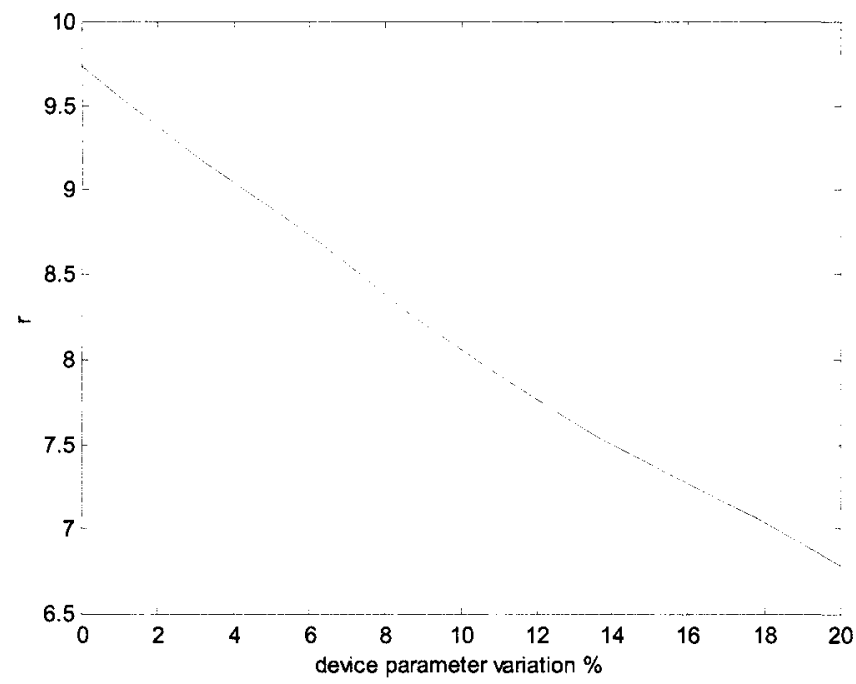

Figure 7. Label detection parameter $r$ versus the parameter $\mathrm{dd}_{0} \mathrm{n}_{\mathrm{s}} /\left(\lambda_{0} \mathrm{R}\right)$ variation.

\section{CONCLUSIONS}

A simple but accurate model for a full E/D that generates/processes $N$ PSK codes simultaneously has been presented. We showed that a standard WGR configuration can be designed as a full $\mathrm{E} / \mathrm{D}$, and we furnish the design guidelines. 
The code cardinality, i.e. the number of PSK codes generated has to be traded for the code processing time. In addition, we show that the code detection parameter increases with $N$ and that it presents a saturation behavior for $N>25$. We also demonstrate that the proposed configuration is quite insensitive to the device parameter variation. A more complete model that takes the actual field distribution inside the waveguides will be the subject of a future work.

\section{REFERENCES}

[1] G. Cincotti, "Full optical encoders/decoders for photonic IP routers", IEEE J. of Lightwave Technol., vol. 22, n. 2, pp. 337-342, 2004.

[2] G. Cincotti, "Design of full optical encoders/decoders for MPLS network", IEEE J. of Lightwave Technol., at press June 2004.

[3] G. Cincotti, "Optical device to generate and process simultaneously optical codes," PCT Patent IT03/000879 filed by University of Roma Tre, December $30^{\text {th }} 2003$.

[4] C. K. Madsen, and J. H. Zhao, Optical filter design and analysis. A signal processing approach, New York: John Wiley and sons inc., 1999.

[5] C. Dragone, C. A. Edwards, and R. C. Kisler, "Integrated optics NXN multiplexer on silicon," IEEE Photon. Technol. Lett., vol. 3, n. 10, pp. 896-899, 1991.

[6] H. Takahashi, S. Suzuki, K. Kato, and I. Nishi, "Arrayed-waveguide grating for wavelength division multi/demultiplexer with nanometer resolution," Electron. Lett., vol. 26, pp. 87-88, 1990.

[7] R. Adar, H. Henry, C. Dragone, R. C. Kistler, and M. A. Milbrodt, "Broad-band array multiplexers made with silica waveguides on silicon," IEEE J. of Lightwave Technol., vol. 11, n. 2, pp. 212-219, 1993.

[8] H. Takahashi, K. Oda, H. Toba, and Y. Inoue, "Transmission characteristics of arrayed waveguide NXN wavelength multiplexer, " IEEE J. of Lightwave Technol., vol. 13, pp. 447-455, 1995.

[9] P. Muñoz, D. Pastor and J. Capmany, "Modeling and design of arrayed waveguide gratings, "IEEE J. of Lightwave Technol., vol. 20, n. 4, pp. 661-674, 2002.

[10] K-i. Kitayama, N. Wada, H. Sotobayashi, "Architectural considerations for photonic IP router based upon optical code correlation," IEEE J. of Lightwave Technol., vol. 18, n. 12, pp. 1834-1844, 2000.

[11] K-i. Kitayama, and N. Wada, H. Sotobayashi, "Photonic IP routing," IEEE Photon. Technol. Lett., vol. 11, n. 12, pp. 1689-1691, 1999. 